\title{
A 2D MEMS Stage for Optical Applications
}

\author{
Caglar Ataman ${ }^{\dagger}$, Yves Petremand ${ }^{*}$, Wilfried Noell ${ }^{*}$, Hakan Urey $^{\dagger}$, Marc Epitaux $^{\dagger}$, Nico F. de Rooij $^{*}$ \\ * Institute of Microtechnology, University of Neuchatel, Neuchatel, Switzerland \\ ${ }^{\dagger}$ Optical Microsystems Laboratory, Koc University, Istanbul, Turkey \\ ${ }^{+}$Intel Corporation, Santa Clara, CA USA \\ cataman@ku.edu.tr
}

\begin{abstract}
A 2D MEMS platform for a microlens scanner application is reported. The platform is fabricated on an SOI wafer with $50 \mu \mathrm{m}$ thick device layer. Entire device is defined with a single etching step on the same layer. Through four S-shaped beams, the device is capable of producing nonlinear 2D motion from linear 1D translation of two pairs of comb actuator sets. The device has a clear aperture of $2 \mathrm{~mm}$ by $2 \mathrm{~mm}$, which is hallowed from the backside for micro-optics assembly. In this paper, a numerical device model and its validation via experimental characterization results are presented. Integration of the micro-optical components with the stage is also discussed. Additionally, a new driving scheme to minimize the settling time of the device in DC operation is explored.
\end{abstract}

Keywords: 2D platform, comb actuator, beam steering, micro-optics

\section{INTRODUCTION}

Micro-optical components integrated on compact MEMS movable platforms enables fast and accurate light manipulation. Thus, such integrated systems have potential applications in diverse fields, such as display and imaging, telecommunications, and particle manipulation.

A 2D MEMS platform designed for a fiber coupling application was previously reported [1]. This paper presents a new platform with the same actuation principle but with a clear aperture of $2 \mathrm{~mm} \times 2 \mathrm{~mm}$, which is being utilized in a microlens scanner array (MLA) based system. The system consists of a single sided MLA, a double sided MLA and a microlens. The double sided MLA and the microlens are required to be moved laterally for the scanning application.

The outline of this paper is as follows: Structural properties and operation principle of the device is presented in section 2. Section 3 presents numerical and experimental results on the performance of the device. Brief information on the scanning system that will employ this platform is given in section 4. Discussions on the micro-optics/MEMS integration and an implementation of an open-loop control system are also included in this section.

\section{THE DEVICE}

\subsection{Device Layout}

A 3D model and an SEM picture of the device are given in Figure 1a and Figure 1b, respectively. The device has four fundamental structural components. The central portion is the hallowed rigid frame designed to hold the micro-optical component to be placed on the stage. The clear aperture of the frame is $2 \mathrm{~mm} \times 2 \mathrm{~mm}$. The actuation of the device is maintained by four set of comb actuators placed on either side of the frame in pairs. For convenience, in Figure 1a, the four comb actuators are named as comb_LR, comb_LL, comb_RL, and comb RR, where $\mathrm{L}$ denotes left and $\mathrm{R}$ denotes right. Movable fingers of the comb actuator pairs on the left and right hand side of the frame are placed on a rigid support beam. The frame and this support beams are connected

MEMS, MOEMS, and Micromachining II, edited by Hakan Ürey, Ayman El-Fatatry,

Proc. of SPIE Vol. 6186, 618603, (2006)

0277-786/06/\$15 - doi: $10.1117 / 12.664777$

Proc. of SPIE Vol. 6186 618603-1 
with each other through four deformable S-shaped mechanical flexure beams. The special design of these beams enables the device to convert 1D linear motion of the comb actuators to 2D nonlinear motion of the frame. The rigid beams carrying the movable fingers are connected to the bulk via four J-shaped beams.

The SOI based fabrication method of the device is straightforward, and this enhances the yield of the process. The platform is fabricated on an SOI wafer with $50 \mu \mathrm{m}$ thick device layer. Entire device is defined with a single etching step on the same layer. A further backside etching step is also required to obtain the hallowed frame.

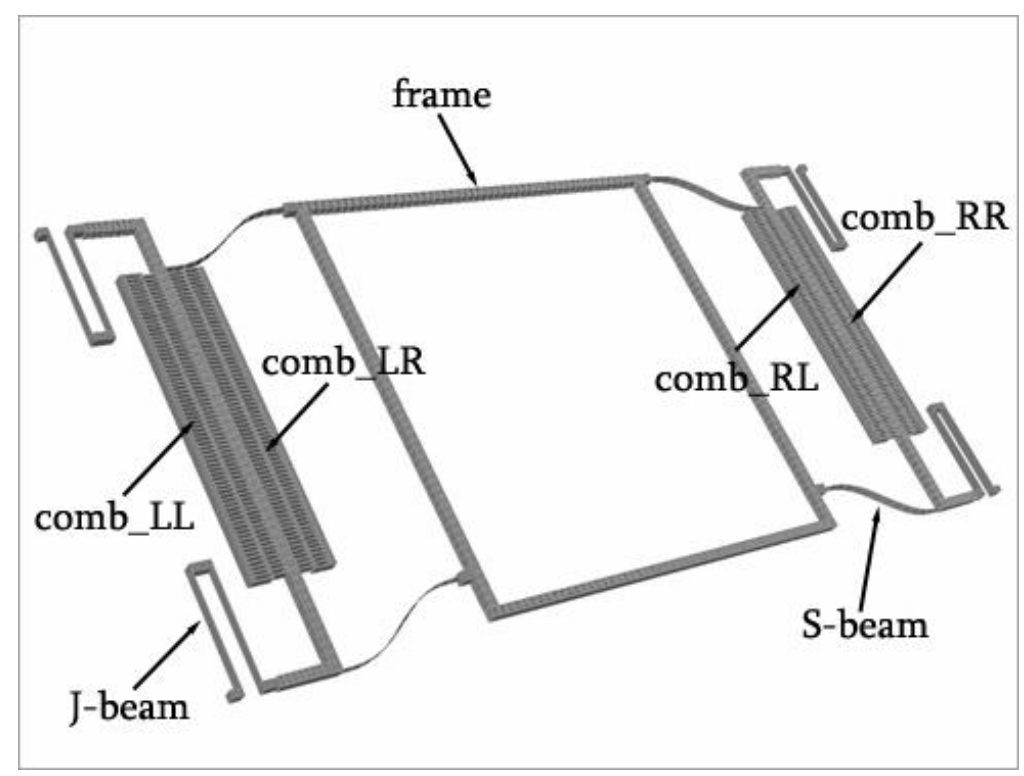

(a)

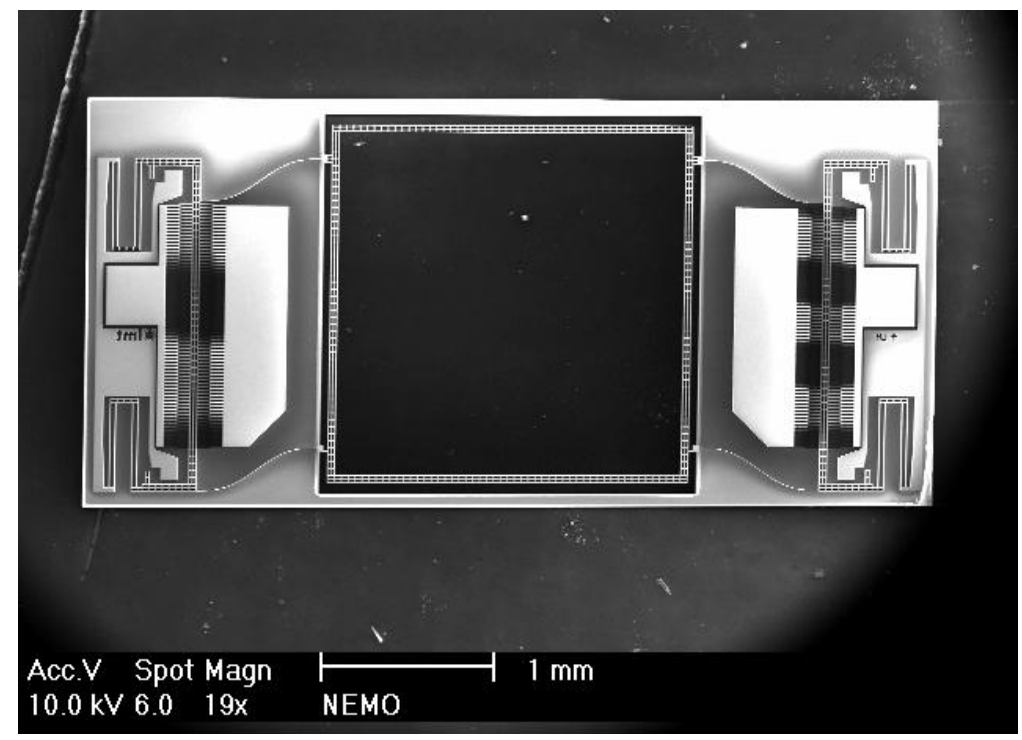

(b)

Figure 1: a) A 3D drawing of the device structure (b) SEM picture of the device

The frame is linked to the actuators by 4 compliant S-beams. These flexible beams are designed to convert two unidirectional $(\mathrm{X})$ actuations into two dimensional $(\mathrm{XY})$ displacements and to amplify the movement in the 
direction orthogonal to the actuators by a factor of two. The curvature of the S-beams is given by the following formula:

$$
y(x)=\frac{\sqrt{2}}{4} x-\frac{\sqrt{2} L}{8 \pi} \sin \left(\frac{2 \pi}{L} x\right)
$$

where, $L$ is the length of the beam in the x-direction. Motion coupling characteristic of the S-beam is shown in Figure 2. If one end of the S-beam is fixed and a unidirectional force in the $\mathrm{x}$ direction is applied to the beam (Figure 2a) from its other end, the tip of the beam moves in both $\mathrm{x}$ and $\mathrm{y}$ directions (Figure 2b).

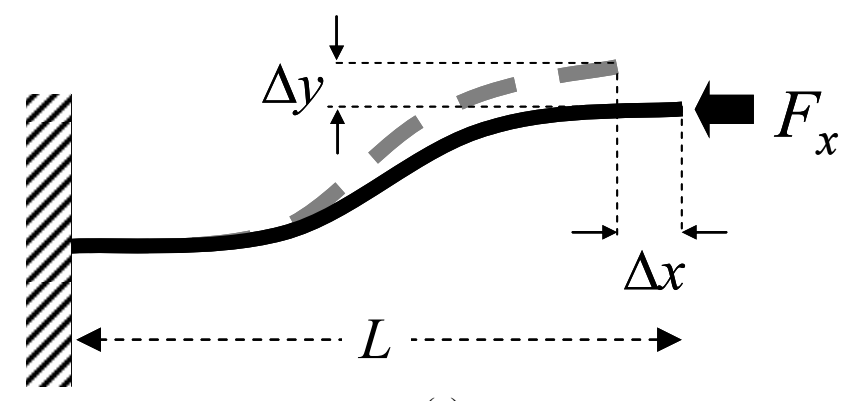

(a)
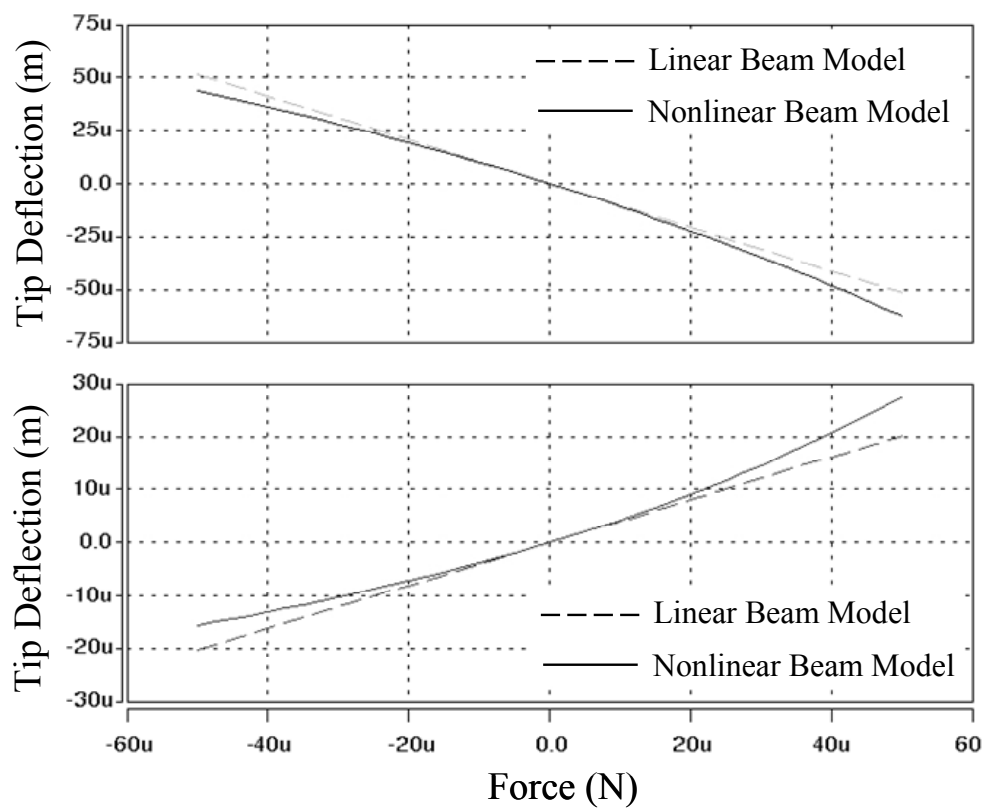

(b)

Figure 2: 2D coupled bending of the S-beam despite the unidirectional applied force. (a) The force is applied on one end of the S-beam in the $x$ direction, while the other end is fixed. (b) Architect ${ }^{\mathbb{B}}$ simulation results for the motion of the free tip of the S-beam in the $\mathrm{x}$ and $\mathrm{y}$ directions.

\subsection{Operation Principle}

The actuator consists of 2 pairs of electrostatic comb drives. When the two actuators are moving together at equal amounts in the same direction (when comb_RR/comb_LR or comb_LL/comb_RL pairs are excited 
together), the platform translates in the $\mathrm{X}$ direction. When the two actuators are moving at equal amounts in opposite directions when (when comb_RR/comb_LL or comb_LR/comb_RL pairs are excited together), the platform translates in the $\mathrm{Y}$ direction by either compressing or extending the S-beams. Addressing of the off-axis points is realized by exciting the individual comb sets in the pairs with different signals amplitudes.

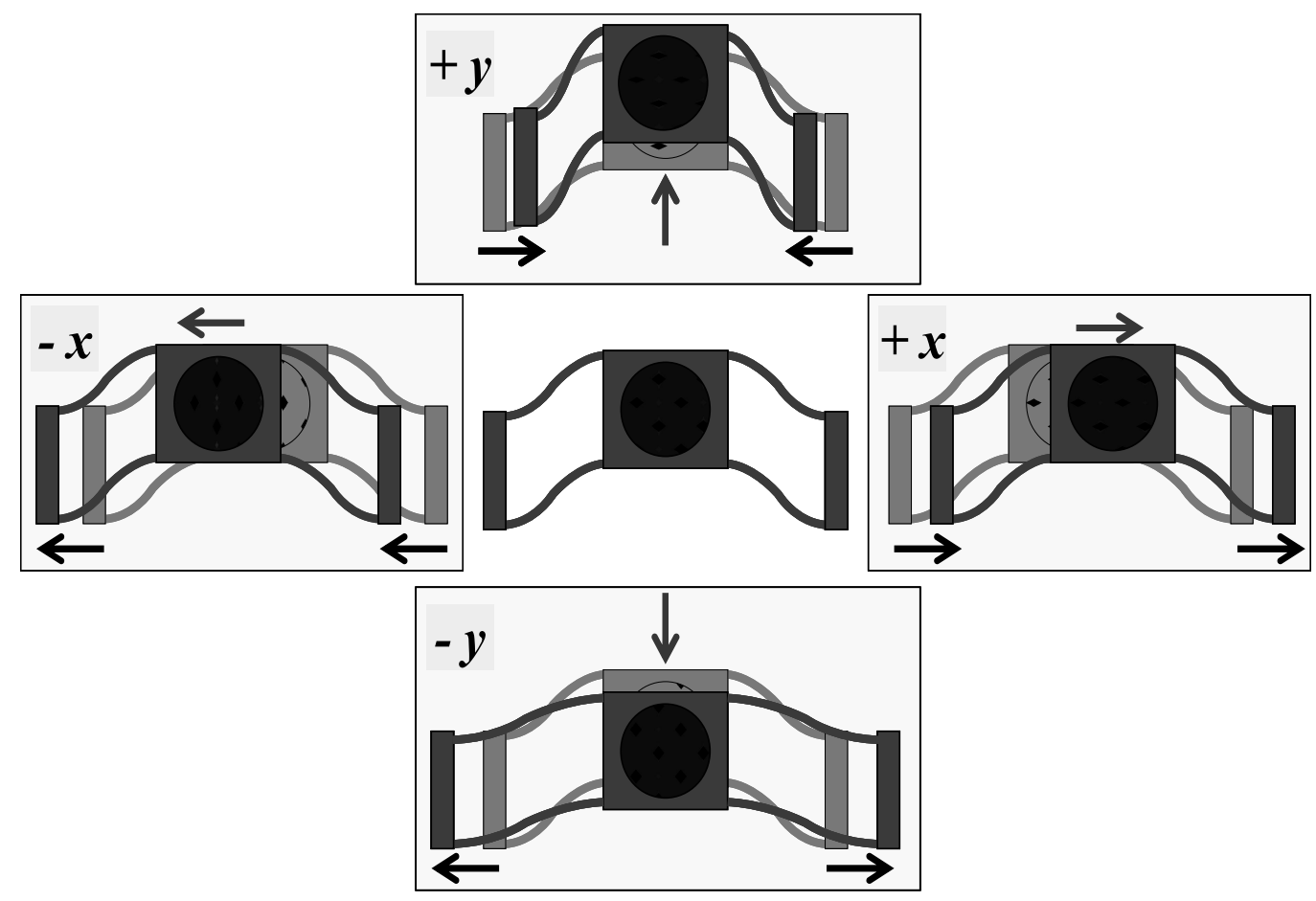

Figure 3: Translational motion of the frame along the horizontal and vertical axes. To move the frame along $\mathrm{x}$ axis, comb_LL/comb_RL or comb_RL/comb_RR pairs should be moved together by equal amounts. In this case the S-beams are neither compressed nor stretched. For motion along y axis, comb_LL/comb_RR or comb_LR/comb_RL pairs should be moved by equal amounts. In this case, the S-beams are either stretched or compressed from both sides by an equal force, leading a unidirectional movement along $\mathrm{y}$.

\section{Numerical and Experimental Results}

A numerical model of the scanning platform was developed using the Architect ${ }^{\mathrm{TM}}$ commercial software. Architect is a system-level simulation tool that enables parametric simulation of static and dynamic behavior of MEMS devices in a fast and reliable way. For further validation of the Architect model, FEA simulations of the structure have been done with ANSYS ${ }^{\mathrm{TM}}$ and Coventor ${ }^{\mathrm{TM}}$ Analyzer. Modal, transient and steady state behavior of the device are simulated with the FEA models and results are compared with the Architect simulations. We present these results here

Simulation results for $\mathrm{x}$ and $\mathrm{y}$ translation of the device versus the excitation amplitude are given in Figure 4, together with the measured displacement data. The comparison of numerical and experimental data show excellent correlation. In the linear region, the displacement of the comb actuators is proportional to the square of the applied voltage. Maximum displacement of the frame was $7 \mu \mathrm{m}$ for $\mathrm{x}$-translation, and $10 \mu \mathrm{m}$ for $\mathrm{y}$-translation both at $80 \mathrm{~V}$. The driving voltage can safely go up to $100 \mathrm{~V}$ which yields a translation of more than $20 \mu \mathrm{m}$ in both directions. 

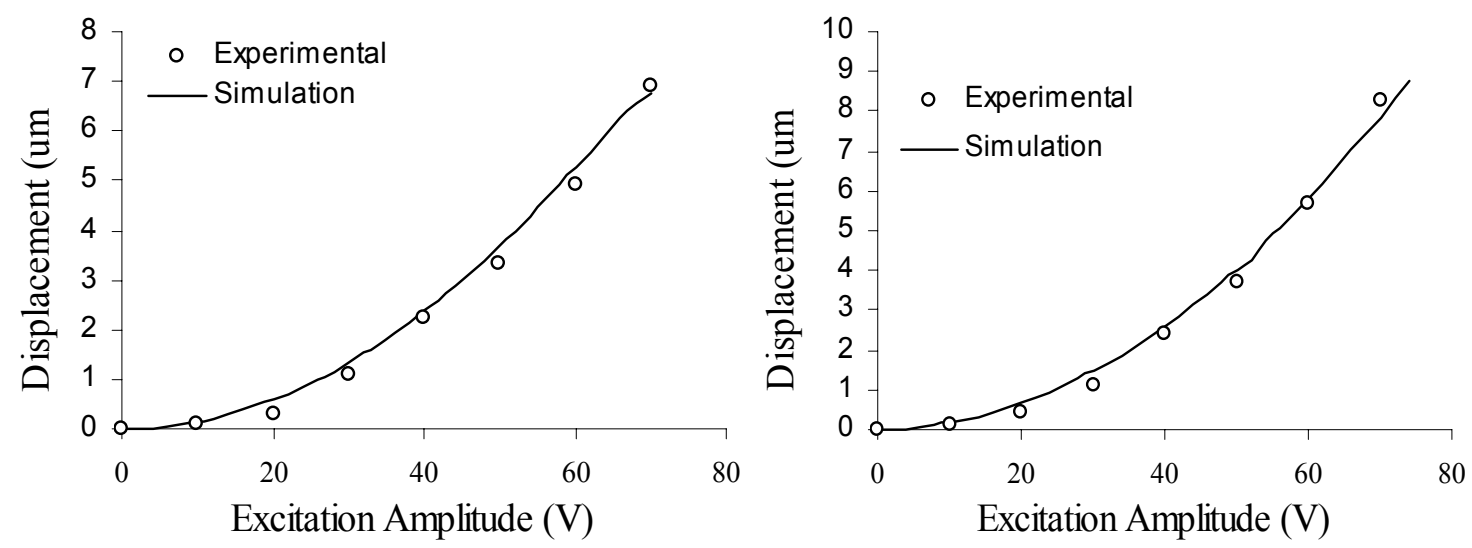

Figure 4: DC performance: (a) Translation along $\mathrm{x}$ axis (b) Translation along $\mathrm{y}$ axis

The modal characteristics of the device are investigated with the ANSYS finite element analysis software. The first five mechanical modes of the device are given in Figure 5.

1st Mode: $829 \mathrm{~Hz}$

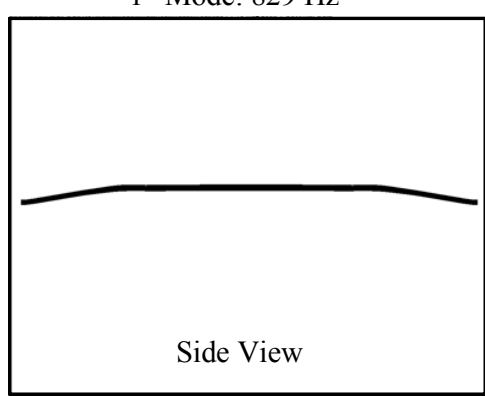

$4^{\text {th }}$ Mode: $2056 \mathrm{~Hz}$

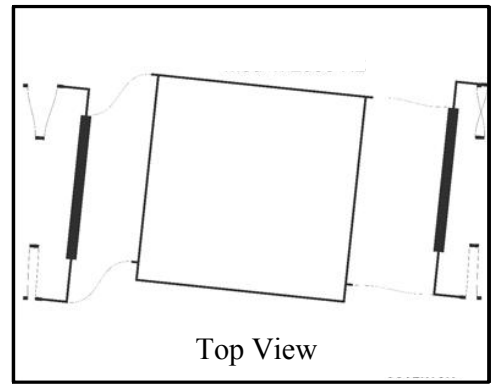

$2^{\text {nd }}$ Mode: $1256 \mathrm{~Hz}$

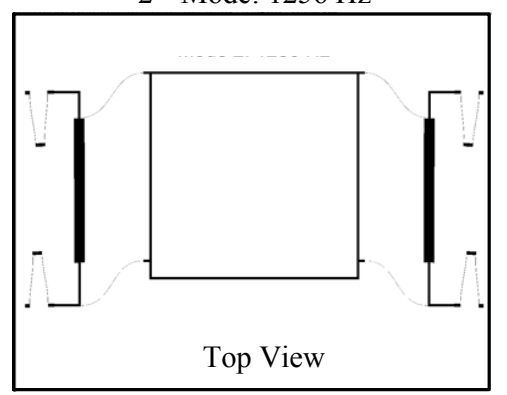

$3^{\text {rd }}$ Mode: $1436 \mathrm{~Hz}$

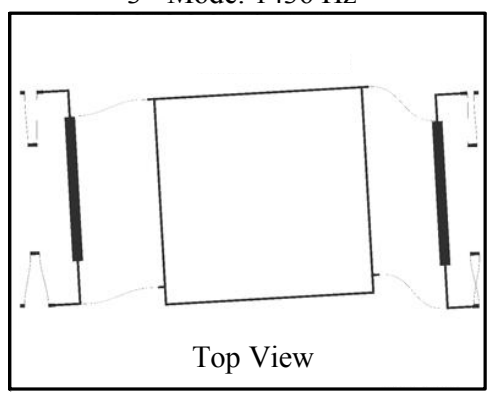

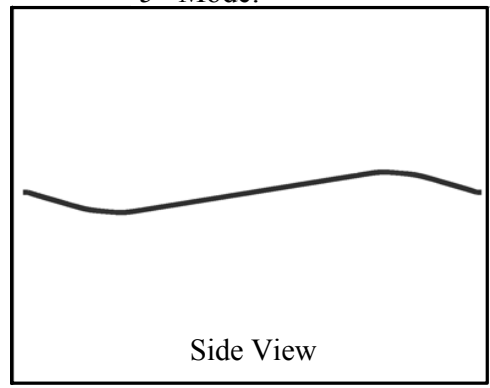

$5^{\text {th }}$ Mode: $3770 \mathrm{~Hz}$

Figure 5: First five mode shapes and associated natural frequencies of the device 


\section{System Integration}

The presented platform is being utilized in a microlens array based beam steering system. An illustration of this scanning system is given in Figure 6. Lateral translation of two MLAs relative to each other allow fast beam deflection, since, even small displacements result in large deflection angles that are due to the small diameters and short focal lengths of the microlenses. Two MLAs separated by a distance of two focal lengths can be used for laser beam steering. In this configuration, the 1st MLA separates the beam into several bundles and focuses each of them onto an intermediate image plane. The 2nd MLA recollimates these bundles and reconstructs the beam. The two main problems with this approach are the spurious light and the discrete addressability of the image plane, which is called the field lens array at the focal plane of the focusing MLA eliminates the spurious light problem [2]. The field lens array and the recollimating MLA can be implemented on the same substrate as a double-sided MLA (DMLA). Without microlens displacement, the field lens array has no effect on the ray bundle; with increasing DMLA displacement, the field lens array bends the ray bundle in the direction of the displacement and makes the chief ray go through the center of the 2nd surface of the DMLA, eliminating the spurious light. The discrete addressability problem arises due to the diffraction effects. Small pitch size and periodicity of the MLAs leads to a discrete diffraction profile. This problem can be solved by the synchronous motion of the DMLA and PSL. Due to the combined motion of the DMLA and the PSL, required displacements are very small; therefore a MEMS platform is a good candidate to obtain this motion. A photograph of the platform carrying a $2.1 \mathrm{~mm} \times 2.1 \mathrm{~mm}$ MLA is shown in Figure 7 . The MLA is thinned down to $300 \mu \mathrm{m}$ to minimize its weight and to prevent the bending of the platform after integration. Preliminary tests of the integrated device showed that the platform can successfully steer the MLA in 2D.

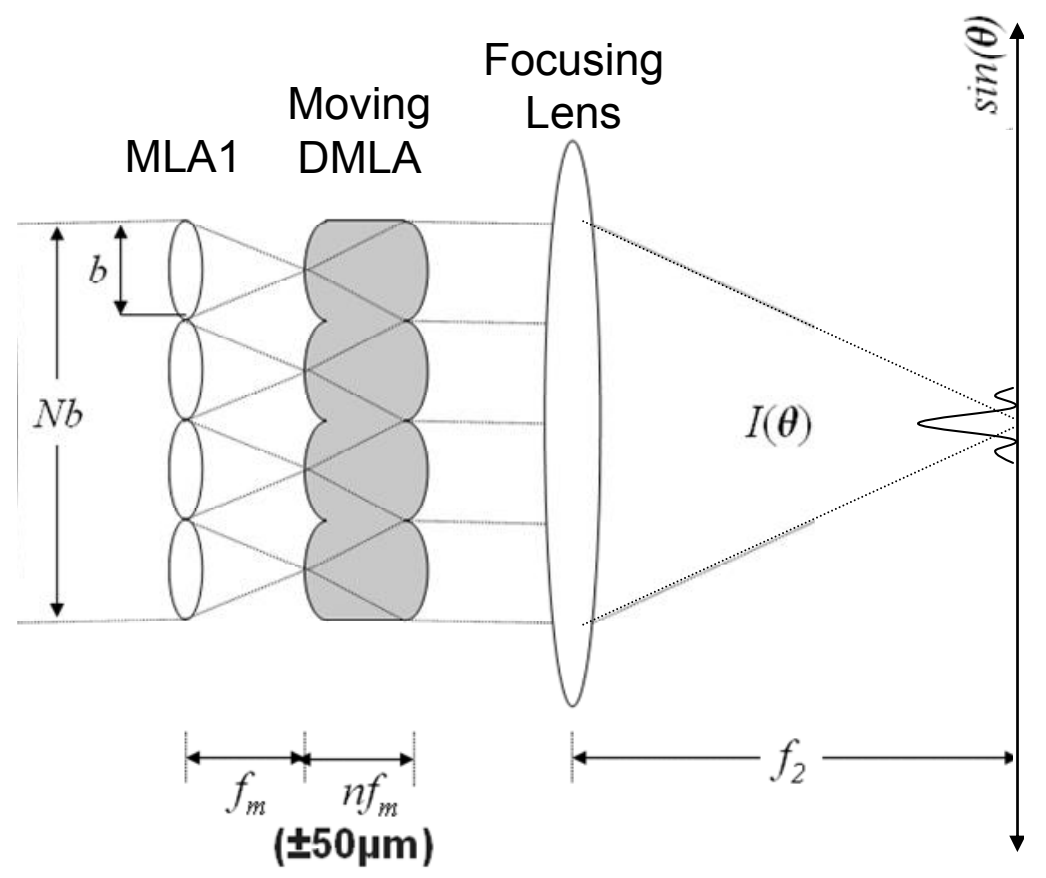

Figure 6: An illustration of the microlens array based scanning system that will utilize the presented platform 


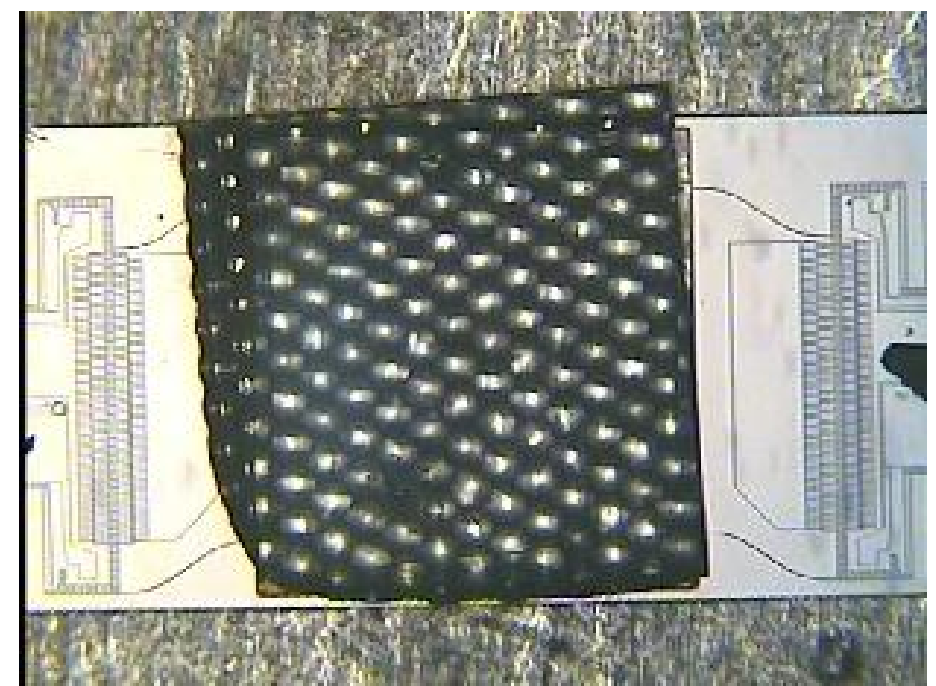

Figure 7: The platform with an MLA integrated on top of it. Alignment of the lens with respect to the frame is not perfect, since the integration is done by hand. However, the integrated device is functional.

The proposed MLA imaging system needs the 2D MEMS platform to perform accurate discrete movements between predetermined positions. For fast operation, the stage should perform this position switching as fast and overshoot-free as possible. Milanovic et.al. from Adriatic Research Institute proposed a new open-loop control method (Inverse Square-Root (ISR) Driving) to minimize the settling time of DC operated MEMS devices [ref]. The method depends on proper pre-filtering the driving signal to remove the frequency components that excites drive resonance. A Simulink model of this open-loop control system is given in Figure 8. The first step of prefiltering of the excitation function is the "System Inverse Filter". Transfer function of this filter is approximately the reciprocal of the mechanical system transfer function; hence it is a band-stop filter that removes out the frequencies around the resonance of the device to avoid overshoot and relaxation oscillations after switching to the new position. In order to make this filter stable and casual, two additional poles should be added to the transfer function. The second step is a Bessel low-pass filter to filter out very high frequencies in the driving signal so that required bandwidth is limited. Due to the voltage Step response of the overall pre-filter for various LPF cut-off frequencies are plotted in Figure 8b. Unlike the initial step function, the pre-filter step response reaches its final value after a certain delay.

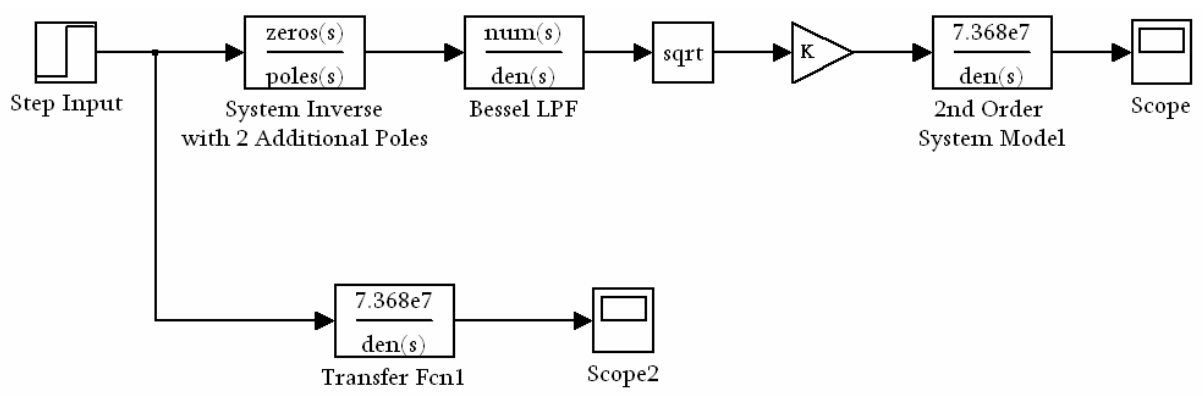

(a) 


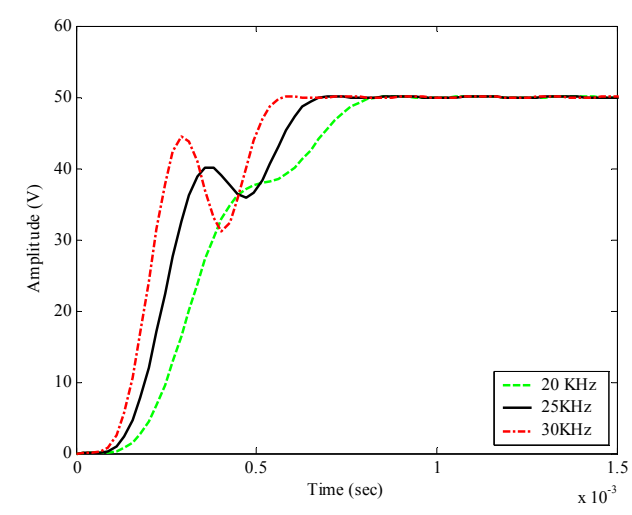

(b)

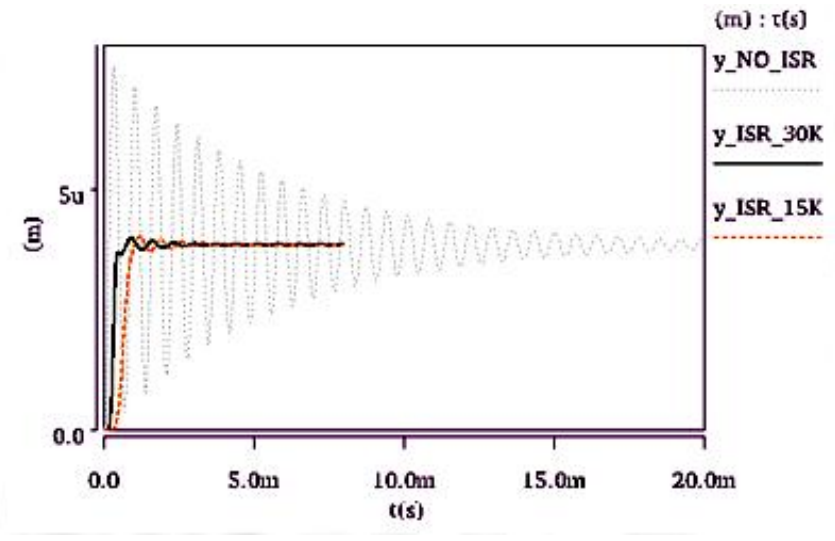

(c)

Figure 8: - ISR driving scheme: (a) Simulink model of the pre-filter (b) Excitation signal for different Bessel filter cut-off frequencies (c) Effect of ISR driving on the step response for Bessel filter cut-off frequency $30 \mathrm{KHz}$ and $15 \mathrm{KHz}$.

To investigate the effect of this open-loop control system on the settling time, a $2^{\text {nd }}$ order model of the device is built using the Architect model. Then the control system is designed using this model and settling times of the device with and without the ISR driving are compared. As given in Figure 8c, pre-filtering of the excitation waveform decreases the settling time of the device drastically. Simulations showed that better settling times can be obtained if the cut-off frequency of the LPF filter is increased; however this also increases the required bandwidth of the electronics.

\section{Conclusions}

A 2D comb actuated MEMS platform for a microlens scanner application is reported. The device is capable of moving more than $20 \mu \mathrm{m}$ in $\mathrm{x}$ and y directions. The platform is fabricated on an SOI wafer with $50 \mu \mathrm{m}$ thick device layer. Entire device is defined with a single etching step on the same layer. 1D motion of the four comb actuator sets can be coupled to enable 2D motion of the device through four S-shaped coupling beams. The characteristics of the se beams are investigated. Experimental and numerical results on the performance of the device are given. The numerical simulations for static and dynamic behavior of the device are performed with Coventor Architect, and they are in very good agreement with the experimental results.

A microlens array based beam scanning application for the designed 2D platform is investigated. For this application, a microlens array is integrated with the platform and steered in 2D. Implementation of an open-loop control system for the platform is also discussed. With this control mechanism, the settling time of the platform can be drastically improved.

\section{Acknowledgements}

The authors would like to thank NEMO (FP6 Network of Excellence in Micro-Optics) for providing partial financial support and Intel Corp. for permission to use 2D MEMS stage technology and partial financial support.

\section{References}

[1] Pétremand, Y. et al, Novel MEMS 2D-Actuator Based on 1D Actuation, Optical MEMS 2004, Proceedings of IEEE/LEOS, Takamatsu, Japan, pp. 58-59 (2004).

[2] J. Duparre, D. Radtke, P. Dannberg, "Implementation of field lens array in beam-deflecting microlens array telescopes," Appl. Opt., 43, 25, 4854-4861, (2004).

[3] V. Milanovic, K. Castelino, „Sub $100 u$ s Settling Time and Low Voltage Operation for Gimbal-less TwoAxis Scanner,“ Proc. of IEEE/LEOS Optical MEMS 2004.

[4] V. Milanovic, K. Castelino, "MEMS-Based High-Speed Low-Power Vector Display" Proc. of IEEE/LEOS Optical MEMS 2005. 